\title{
KAREN WAREN: LA ÉTICA ECOFEMINISTA
}

\author{
KAREN WAREN: THE ECOFEMINIST ETHICS
}

Diana Paola Triana Moreno*

Recebido: 09/2016 Aprovado: 05/2017

\begin{abstract}
Resumen: El ecofeminismo social de Karen Warren es una de las aproximaciones más sólidas a una nueva comprensión de la ética medio ambiental. Su categoría analítica 'lógica de la dominación' permite articular la teoría feminista y ecológica en los debates éticos contemporáneos, cuestionando el antropocentrismo y postulando una versión renovada de valores, principios y agentes morales. El ecofeminismo de Warren es vital no sólo para comprender la subordinación de las mujeres, los animales y la tierra, sino para el desarrollo de teorizaciones y prácticas ecológicamente responsables y socialmente justas para todos. Este artículo de reflexión tiene como objetivo efectuar una aproximación al ecofeminismo de Karen Warren explicando qué son la lógica de la dominación y los marcos conceptuales opresivos, además de identificar cuál es la alternativa ética que postula y sus limitaciones.
\end{abstract}

Palabra clave: Ética, ecofeminismo, ecología, medio ambiente, feminismo

\begin{abstract}
The social ecofeminism of Karen Warren is one of the strongest to a new understanding of environmental ethics approaches. Her analytical category 'logic of domination' can articulate feminist and ecological theory in ethical contemporary discussions, questioning anthropocentrism and postulating a revamped version of values, principles and moral agents. Warren ecofeminism is vital not only to understand the subordination of women, animals and earth, but for the development of theorizing and ecologically responsible and socially fair to all community. This article aims reflection make an approach to eco-feminism Karen Warren explaining what the logic of domination and conceptual frameworks are oppressive, and identify what is the alternative ethic that postulates and limitations.
\end{abstract}

Keyword: Ethics, ecofeminism, ecology, environment, feminism

\section{Introducción}

Desde la década de 1980 la interconexión entre filosofía, feminismo y ecología ha permitido la aparición de nuevos problemas y reflexiones donde la perspectiva ecofeminista ha tenido una presencia importante. Sin duda el ecofeminismo aparece tratando de solucionar una serie de obstáculos que presentaban tanto los feminismos como las teorías ecologistas durante la década del 1970, en el sentido en que las primeras se ubicaban desde una perspectiva antropocéntrica y las segunda resultaban androcéntricas (Ruether, 2001; Gómez, 2010; Triana, 2016). Solo con la aparición del ecofeminismo se identifican una serie de coincidencias entre las luchas de ambos movimientos revelando que más allá de sus particularidades existía un sistema jerárquico y opresivo que mantenía una gemela dominación entre la naturaleza y las mujeres, de modo que se reconoce que en el fondo de los dos movimientos debe existir un apoyo mutuo que permita prácticas éticas al margen de cualquier sistema de dominación (Warren e Cheney, 1991; Warren, 2003).

El ecofeminismo se presenta como una forma de feminismo

\footnotetext{
* Master en Estudios Sociales de la Universidad Pedagógica Nacional. Filosofa de la Universidad Libre e historiadora de la Universidad del Rosario. Actualmente es becaria del Doctorado en Filosofía de la Pontificia Universidad Javeriana
}

Problemata: R. Intern. Fil. V. 8. n. 2 (2017), p. 158-168 ISSN 2236-8612 doi:http://dx.doi.org/10.7443/problemata.v8i2.30756 
alternativo y como una forma de ética ambiental (Warren, 2003), que se ocupa de estudiar la dominación de la mujer y otros grupos oprimidos en vínculo con la dominación de la naturaleza. No obstante, es preciso señalar que el ecofeminismo ha adquirido diversas interpretaciones alrededor de la interconexión entre las mujeres y la naturaleza, manifiestas en al menos dos posturas desde el punto de vista ético: el ecofeminismo social y el ecofeminismo espiritualista (Triana, 2016). En ambos casos, el feminismo ecológico puede y propone "ofrecer un marco distintivo para reconcebir el feminismo y desarrollar la ética medio ambiental, de tal modo que las conexiones entre la dominación de la mujer y la dominación de la naturaleza sean seriamente consideradas"(Warren, 2003).

La aproximación del ecofeminismo social considera que la conexión mujer-naturaleza es producto de una construcción social patriarcal que procura justificar la propiedad y utilización de las mujeres y el mundo natural en conjunto bajo un mismo argumento de dominio (Ruether, 2001; Glazebrook, 2002; Warren, 2003). En ese sentido, parte de la iniciativa de esta tendencia está enfocada en deconstruir los dualismos que han soportado la lógica de la dominación con el propósito de integrar al conjunto de los seres humanos con la naturaleza, evitando perpetuar la comprensión que feminiza la naturaleza y naturaliza a las mujeres (Glazebrook, 2002; Warren, 2003).

La propuesta de la filósofa norteamericana Karen Warren, desde una perspectiva social, política y filosófica, es una de las aproximaciones más sólidas a esta nueva vía interpretativa. Su categoría analítica 'lógica de la dominación' permite articular la teoría feminista y ecológica en los debates éticos contemporáneos en torno a la ética ambiental, cuestionando el antropocentrismo y androcentrismo y postulando una versión renovada de valores, principios y agentes morales. El ecofeminismo de Warren es vital no sólo para comprender la subordinación de las mujeres, los animales y la tierra, sino para el desarrollo de teorizaciones y prácticas ecológicamente responsables y socialmente justas para todos (Warren, 2003).

Este articulo tiene como objetivo efectuar una aproximación al ecofeminismo de Karen Warren explicando qué son la lógica de la dominación y los marcos conceptuales opresivos, además de identificar cuál es la alternativa ética que postula y revelar algunas de las críticas que se le han efectuado desde diferentes perspectivas.

\section{Lógica de la dominación}

El interés del ecofeminismo es eliminar todos aquellos factores que 
contribuyen a la dominación, subordinación y explotación sistemática de la mujer y la naturaleza. Para Warren el asunto debe ser comprendido desde la misma conceptualización de las categorías analíticas que conforman la gemela dominación y que se ubican como un conjunto argumentativo falaz que termina justificando una situación injusta y claramente indeseable.

De acuerdo con la autora, existen marcos conceptuales que permiten efectuar una taxonomía del mundo en el que vivimos, los cuales determinan nuestra experiencia histórica y culturalmente compartida y modelan la forma en que los sujetos adecuan sus experiencias de mundo. En ese sentido, los marcos conceptuales están sedimentados en las creencias, valores, actitudes y expectativas que existe entre los sujetos en un entramado históricamente situado. Esto permite tener una mirada del mundo socialmente construida que determina la manera en que nos percibimos a nosotros mismo y los demás, donde se entrecruzan cuestiones raciales, de género, etnia, edad, clase, entre otras (Warren, 2003).

Ahora bien, los marcos conceptuales son absolutamente necesarios para ser en el mundo conforme lo enunciado por Warren, el problema aparece cuando esos marcos conceptuales se convierten en opresivos, es decir que explican, justifican y mantienen condiciones de dominación y subordinación. De ahí que el argumento opresivo implica un pensamiento de valores jerárquico, dualismos de valor -antagónicos y exclusivos- y la lógica de la dominación. Estas tres características conforman los marcos conceptuales opresivos, pero sólo la última corresponde a la estructura básica de la argumentación que justifica el dominio, por ello Warren señala en su ensayo "El poder y la propuesta del ecofeminismo" que esta última es la característica más significativa.

La lógica de la dominación requiere de un sistema de valores que pueda soportar y justificar la dominación en sí misma y permita considerar que la subordinación es 'justa'. Cuando se establece una jerarquía de valor se suele considerar per se que existe uno preferible que debe privilegiarse sobre otros miembros de la jerarquía.

Si pensamos en la jerarquía por sí sola o en los dualismos de valor no obtendremos más que diferencias dentro de una clasificación tal como ocurre con la escala cromática donde cada color le corresponde un lugar en relación con los otros del conjunto, pero eso no lo hace preferible o más apreciado que los demás. Las similitudes y diferencias no son en ningún sentido formas de garantizar la subordinación, lo que las transforma es el trasfondo ético que establece la lógica de la dominación. Así, cuando el argumento de la subordinación remete a considerar lo 'justa' que ésta resulta, se está necesariamente haciendo referencia a un horizonte ético en el que 
determinamos como justo y deseable un modelo sistémico de dominio. En consecuencia con esta forma de deconstrucción, el ecofeminismo de Warren intenta eliminar cualquier esquema de dominio o 'ismo' -sexismo, heterosexismo, racismo, clasismo, etc.-

La lógica de la dominación ha operado históricamente desde el patriarcado para mantener y justificar la dominación gemela de la naturaleza y las mujeres, de modo que todo feminismo -no solamente ecofeminista- debe promover el desmonte de esta forma de comprensión (Warren, 2003). En este sentido, la lógica de la dominación y cualquier marco conceptual que genere esta lógica deben ser eliminados para acceder a relaciones que revelen las diferencias sin que con ello se sedimenten éticas que alienten los esquemas jerárquicos y de dominio.

\section{La revolución ética del ecofeminismo}

El ecofeminismo tiene una doble responsabilidad dentro de la revolución de la ética, por un lado, debe criticar el sexismo oculto en algunas tradiciones éticas y, por otro lado, formular una ética que no reproduzca la lógica de la dominación. Pero, ¿cómo pensar una ética que renuncie a la lógica de la dominación? o, ¿cuáles son las características de una ética en clave feminista? Para ello, es necesario señalar por qué todo feminismo debe ser ecofeminismo, luego, analizar en primera instancia cuál es la discusión del ecofeminismo de Warren en referencia a las tradicionales éticas occidentales; $y$, finalmente, establecer los planteamientos centrales para de una ética ecofeminista.

En primer lugar, los feminismos hasta la década de 1980

1, no habían avizorado la conexión en la lógica de la dominación que existía entre las mujeres y la naturaleza, motivo por el cual los discursos se orientaban a localizar las raíces conceptuales de la opresión sexista dentro del orden patriarcal, una tarea que fue fundamental. En buena medida, las críticas de las feministas al patriarcado ignoraba todo aquello que estaba por fuera de las relaciones humanas, dejando de lado en sus debates y luchas el lugar central que ocupa el medio ambiente y los animales, al privilegiar una perspectiva crítica al sexismo. Entonces, el naturismo entendido como lógica de la dominación y justificación de superioridad de los seres humanos sobre la naturaleza es central para comprender el giro que introduce la ética ecofeminista. Cuando se cuestiona la lógica de la dominación y se comprenden que todas las formas de opresión sexual, étnica, racial y clase, mantienen el su fundamento el mismo argumento, entonces se debe asumir que existe un 
núcleo argumentativo que se debe rechazar desde cada una de estas diferentes perspectivas (Warren, 2003). De modo que el feminismo tendría que rechazar con igual vehemencia el marco conceptual opresivo sexista, de la misma manera que los otros "ismos", entre ellos el naturismo.

Igualmente, el compromiso del ecofeminismo por reconcebir el feminismo está vinculado con la necesidad de enfatizar en la centralidad del dominio de la naturaleza. Por ello, Warren afirma que la apertura a la categoría de género fue vital para comprender la historicidad de las categorías y, por con ello, el ecofeminismo va a insistir en la construcción social de la naturaleza. Si se asume la naturaleza como una categoría socialmente construida, histórica y culturalmente situada, entonces es posible rastrear cómo hay unas coincidencias en las lógicas de la dominación que hacen gemela la subordinación de las mujeres y la naturaleza (Puleo, 2011). Empero, esto no significa negar la materialidad de los árboles, ríos, plantas y animales, sino simplemente enuncia que la forma en que los consideramos como parte del entramado ético está determinada en buena medida por la forma en que les comprendemos:

\footnotetext{
"en cualquier discusión sobre la «opresión y dominación de la naturaleza» hay que referirse a las formas especificas en que los humanos han dominado la naturaleza a lo largo de la historia; y en cualquier discusión sobre la «dominación de la mujer» hay que referirse a las formas especificas en que el hombre ha dominado a la mujer a lo largo de la historia"(Warren, 2003)
}

En este sentido, el ecofeminismo debe comprometerse con un feminismo abiertamente vinculado con una ética medioambiental, a través de la desarticulación de los esquemas binarios y antropocéntricos. Por esta razón el ecofeminismo supone un distanciamiento con las éticas tradicionales en Occidente, en la medida en que éstas tienen una perspectiva exclusiva de los seres humanos como sujetos morales, puesto que solo los agentes morales humanos pueden satisfacer la noción de responsabilidad y deliberación indispensables para la ética. Las éticas deontológicas y utilitaristas buscan satisfacer las necesidades de la comunidad moral humana y no dan lugar a la incorporación de otros agentes morales, como si se efectúa desde la perspectiva de la ecología profunda (Glazebrook, 2002). El antropocentrismo de los presupuestos éticos tradicionales reposa en la lógica de la dominación naturista, al considerar que solo cuentan moralmente los seres humanos y desconocen la posibilidad de ampliar los límites de sus presupuestos, conforme a las propuestas ya desarrolladas desde el ecocentrismo y biocentrismo, presentes en las visiones éticas del ecologismo y el ambientalismo (Gandon, 2009; Gómez, 2010; Triana, 2016).

Entonces, la propuesta de Warren presenta al menos tres elementos 
centrales para formular una ética más justa para toda la comunidad de la vida: la narrativa, la afectividad, y la relación igualdad/diferencia justa y jerárquica.

Para Warren hay que considerar la forma de construir narraciones éticas que no necesariamente remitan a argumentos lógicos, sino que permitan situarse en una perspectiva que revalore el ámbito sentimental de las relaciones. La narración en primera persona es un recurso literario para efectuar relatos sobre las experiencias personales. Esta manera de describir las experiencias permite articular a los discursos el universo sentimental y reconocerse subjetivamente implicado por los otros, esto es, «estar en relación con» otros, incluyendo humanos y no humanos. En este sentido, para Warren la existencia de una relación moral solo supone que al menos uno de los implicados sea un agente moral, en tanto que la ética se situaría en el reconocimiento de las relaciones vitales con otros y sus interdependencias.

Asimismo, la narrativa en primera persona expresa una variedad de actitudes y comportamientos éticos, que han estado marginadas de las éticas tradicionales en Occidente. Para la autora existe una diferencia radical entre el comportamiento entre dos manera de narrar la relación con la naturaleza: dominación y conquista o respeto y afectividad. Cuando nos enfrentamos a la situación de escalar la montaña podemos narrar el desafío de maneras muy diversas y el énfasis que hacemos en las expresiones que se usan cambian radicalmente la posicionalidad ética del narrador: escalé con determinación, fuerza y conocimiento para llegar a la cima y lograr cumplir el fin que era estar sobre la montaña; en contraste, tomé un bocanada de aire, escuché con atención, sentí la roca frente a mí y me serené, descubrí la sensación de estar en relación con la naturaleza y ese era el único fin (Warren, 2003).

En fondo de la propuesta de Warren, integrar el feminismo a la ética medio ambiental ofrece la posibilidad de concebir éticas que emergan a partir de casos concretos, a partir de las situaciones particulares en las que se entra "en relación con» otro, lo cual implica un distanciamiento de las éticas procedimentales y prescriptivas, pues ya no abrían principios o normas predeterminadas y abstractas. De esta manera, Warren concibe la ética como una construcción social, histórica y cultural, siempre abierta y en formulación: "el uso de la narrativa en la ética, como una reflexión de las experiencias vividas y sentidas, produce una postura en la que el discurso ético puede contener las realidades sociales, históricas y materiales en las que los sujetos morales se encuentran"(Warren, 2003). Por eso la ética debe integrar las múltiples voces, que pongan en juego un amplio horizonte de actitudes, comportamientos, creencias y valores alrededor de la formulación de un discurso ético afectuoso:

«La percepción afectuosa» supone y mantiene la diferencia -la distinción entre uno y 
otro, entre el humano y el no humano- de tal modo, que la percepción del otro como ajeno es una expresión de afecto por algo o alguien que, desde el comienzo, es reconocido como independiente, diferente, distinto (...) «La percepción» afectuosa del mundo natural no humano es una intento por entender qué quiere decir para los humanos respetar el mundo no humano"(Warren, 2003).

En este sentido, las éticas tradicionales son asociadas por Warren no solo con la actitud de conquista y control, sino sobre todo con los marcos conceptuales opresivos en relación a las mujeres y a la naturaleza. El sistema patriarcal ha consolidados un marco conceptual opresivo que está a la base de los postulados éticos tradicionales, dando como resultado en las éticas deontológicas la idea de sujetos universales y principios abstracto que son antropocéntricos y androcéntricos y, desde el utilitarismo el encubrimiento de condiciones histórico culturales injustas en referencia a las mujeres y al mundo natural que justifican la subordinación. Así para el ecofeminismo social no se puede hablar de la mujer universal, sino de mujeres en plural con características singulares social e históricamente situadas, afirmando una ética articulada por diferencia e interrelacionalidad (Warren, 1999; Glazebrook, 2002; Warren, 2003; Sagols, 2014).

En consecuencia, no es posible desde la óptica de Warren mantener éticas de carácter universal que niegan la posicionalidad y la historicidad, sin que ello signifique que su postura sea favorable con un mero relativismo ético. Para la autora es una apuesta por la construcción de un "universal situado", es decir, una búsqueda permanente por la universalidad de la ética, sin perder de vista su transformación permanente y su carácter de flexible y abierta. Sin duda el relativismo se presenta como una amenaza que debe ser superada, porque afirmar que la ética es parcial no es renunciar a construir modos más justos e igualitarios para los miembros de la comunidad moral. Justamente, como resultado de la historicidad de lo ético, es que aparece la ética ambiental y los discursos ecológicos que dan cuenta de una crisis profunda en la relación con la naturaleza.

Esto conduce a analizar, en último lugar, la relación igualdad/diferencia justa y jerárquica en la propuesta de Warren. La crítica a las éticas antropocéntricas y androcéntricas como vía para asegurar un distanciamiento de la lógica de la dominación, supone concebir una ética feminista y ecocéntrica. Por ello, la ética de Warren afirma la interdependencia entre los humanos y no humanos, encaminada hacia una versión inclusiva e interrelacional pero no igualitaria, en el sentido de considerar que todos merecen un mismo tratamiento en el universo moral. Eso significa que hay una igualdad básica en la medida en que todos (aguas, plantas, animales, humanos) hacen parte de la comunidad moral, pero no tienen dentro de ella el mismo estatus, razón por la cual se trata de una jerarquía: "somos iguales y 
diferentes".

En este sentido, Warren va a retomar la perspectiva ecológica de Aldo Leopold (2004) al asegurar una jerarquía entre los miembros de la comunidad moral, ya no en un sentido de conquistador, sino como un simple miembro de ella. El asunto para Warren está en que establecer un sistema ético jerárquico no implica afirmar la lógica de la dominación, puesto que una cosa es la jerarquía y otra el marco conceptual opresivo, este esquema no permite justificar la explotación, dominio o control sobre los otros: "sí hay una superioridad del ser humano, pues no podemos negar que estamos mejor equipados que las rocas y las plantas para reformar de modo radical el entorno en modos conscientes de autodeterminación"(Sagols, 2014). La diferencia y la jerarquía por si solas no se configuran como un marco conceptual opresivo en tanto no garantizan las subordinación de unos por otros y mucho menos una valoración moral sobre la justicia.

Para finalizar, intentaré dar cuenta de una de las críticas que ha recibido el ecofeminismo social de Warren. Si la naturaleza como conjunto incluye en ella a las rocas, las plantas, los animales, esto es a los no-humanos, tenemos que cuestionar el lugar que supone pensar la jerarquía de valor, con independencia de la lógica de dominación. ¿Qué pasa con los animales en la teoría ecofeminista de Warren? Al parecer la teoría de la jerarquía ecológica, permite considerar que en medio de las interacciones de los sistemas bióticos es preciso, deseable y necesario el hecho de contribuir a la cadena alimenticia del conjunto. En ese sentido, todo debemos hacer una contribución: "es necesario que ofrezcas esta bendición al cuadrúpedo y que a tu debido tiempo, respondas con tu cuerpo de esta manera, ya que el cuadrúpedo da su vida para tu supervivencia"(Warren, 2003). Sin embargo, esta mirada sobre los animales los instrumentaliza y les resitúa en una condición de subordinación justa dentro de la jerarquía de valor conforme a la lógica de opresión especista. Así, los animales no cuentan como seres vivos iguales, seres vivos sensibles, o sujetos de derechos, sino que hacen parte de las condiciones de supervivencia de la vida humana y fomenta su explotación.

Warren cuestionaría no el asunto de alimentarnos con los otros miembros de la comunidad moral, sino lo relativo a las condiciones de bienestar en los que se encuentran los animales. De modo que la pregunta moral no hace referencia a si comemos o no carne, sino qué tipo de carne comemos y bajo qué condiciones se produjo. Esto encubre una problema mayor relacionado con las crisis ambiental producto de la exploración animal para fines de consumo humano.

Por eso para Carol Adams (Adams, 1991) la perspectiva de Warren presenta serios problemas al momento de reconocer que el ecofeminismo que 
ella plantea, al estar situado dentro de la cultura, fomenta el consumo de carne, desconoce los costes medio ambientales del consumo, instrumentaliza a los animales, y por lo tanto, separa las consecuencias del consumo de carne con la experiencia misma de comerla. De modo que no sería el amor, el respeto y la reciprocidad los soportes sentimentales de una nueva ética que más allá de superar la lógica de la dominación naturista y sexista, termina por instalarse cómodamente en el especismo, a pesar de sus iniciativas. Los animales vistos fuera de la lógica de la dominación debe acabar con la opresión de los mismos "esta identificación significa una redefinición de la relación con los animales, que dejan de ser instrumentos, medios para nuestros fines, y se convierten en seres que merecen vivir, y a los que respetamos sin necesidad de tener que entablar amistad con ellos" (Adams, 1991)

\section{Conclusión}

El ecofeminismo está comprometido con una reformulación del feminismo, en la medida en que se propone incorporar a las teorizaciones, discusiones y luchas feministas el dominio de la naturaleza como otro tema central de la agenda feminista. En el fondo hay una propuesta que busca una reformulación de la ética medioambiental que desarticula y discute con la tradición ética occidental, específicamente, utilitarista y deontológica. Inclusive, la perspectiva de Warren es implacable con el reduccionismo o neutralidad valorativas que suponen las éticas tradicionales, en tanto se distancia de los dualismos razón/emoción, mente/cuerpo, y la naturaleza/cultura. Finalmente, el ecofeminismo, de acuerdo con Karen Warren, ofrece un marco para la formación de una ética distintiva, feminista y ecocéntrica. Con ello, el ecofeminismo se aspira avizorar un punto de vista que supere los marcos conceptuales opresivos con una decisión radical de transformar las relaciones éticas de cara a construir un horizonte de futuro más justo para vivir, esto es, la revolución ética ecofeminista.

\section{Bibliografía}

ADAMS, C. Ecofeminism and the eating of animals. Hypatia, v. 6, n. 1, p. 125, //Spring91 1991. ISSN 08875367. Disponível em: < http://ezproxy.uniandes.edu.co:8080/login?url=http://search.ebscohost.com/logi

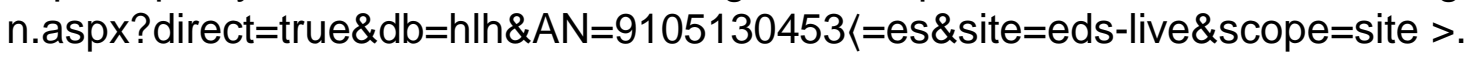


GANDON, A.-L. L'écoféminisme: une pensée féministe de la nature et de la société. (French). Ecofeminism: Feminist Theories of Nature and Society. (English), v. 22, n. 1, p. 5, 06// 2009. ISSN 08384479. Disponível em: < http://ez.urosario.edu.co/login?url=http://search.ebscohost.com/login.aspx?direc $\mathrm{t}=$ true $\& \mathrm{db}=$ edo\&AN=42311504〈=es\&site=eds-live $>$.

GLAZEBROOK, T. Karen Warren's ecofeminism. Ethics \&amp; the Environment, v. 7, n. 2, p. 12, 2002. ISSN 10856633. Disponível em: < http://search.ebscohost.com/login.aspx?direct=true\&amp;amp;db=eih\&amp;am p;AN=7733400\&amp;amp;lang=es\&amp;amp;site=eds-live >.

GÓMEZ, L. F. Fundamentos de ecoética. Académica colombiana de ciencias veterinarias, v. 2, p. 11-31, 2010. ISSN 22159800. Acesso em: 12 Agosto 2014.

LEOPOLD, A. La ética de la tierra. In: VALDÉS, M. (Ed.). Naturaleza y valor. México: Fondo de cultura económica, 2004. p.25-44.

PULEO, A. Ecofeminismo para otro mundo posible. Madrid: Cátedra, 2011. 439.

RUETHER, R. R. Deep Ecology and World Religions : New Essays on Sacred Grounds. In: (Ed.). SUNY Series in Radical Social and Political Theory. New York: State University of New York Press, 2001. ISBN 9780791448830 9780791491058.

SAGOLS, L. El ecofeminismo y su expresión en la filosofia de Karen Warren. Una perspectiva ética. Debate Feminista, v. 49, n. 25, p. 116-124, 2014. ISSN 01889478. em: http://search.ebscohost.com/login.aspx?direct=true\&db=a9h\&AN=96319869/=e s\&site=eds-live $>$.

TRIANA, D. Éticas ecofeministas: la comunidad de la vida. Cuadernos de Filosofía Latinoamericana, v. 37, p. 117-131, 2016. ISSN ISSN 0120-8462.

WARREN, K. Chapter 8: Care-sensitive ethics and situated universalism. In: (Ed.): Taylor \& Francis Ltd / Books, 1999. p.131-145. ISBN 9780415197366.

Filosofías ecofeministas. Barcelona (España) Icara 2003, 2003. ISBN 8474266831. Disponível em: < http://ez.urosario.edu.co/login?url=http://se... >. 
WARREN, K. J.; CHENEY, J. Ecological Feminism and Ecosystem Ecology. Hypatia, v. 6, n. 1, p. 179-197, 1991. ISSN 08875367. Disponível em: < http://www.jstor.org/stable/3810040 >.

1 Feminismos liberales, tradicional, marxista, radical, socialista, de mujeres negras y no occidentales, lesbofeministas propendían por acabar con el sexismo y el orden patriarcal, sin considerar las conexiones con la ecología y el ambientalismo (Warren, 2003). Sin embargo, es necesario señalar que la tradición del giro ecológico del feminismo se inició con François D'Eaubonne en la década de 1970. Esta última perspectiva, se mantuvo por fuera de la tradición feminista y de su clasificación en olas. D'Eaubonne señaló que era necesario pensar en la justicia y la igualdad entre todos los seres vivos y ecosistemas, denominado ethos feminista ecoético (Sagols, 2014). 